\title{
LAS PINTURAS MURALES DE JUAN MIGUEL SÁNCHEZ EN LA CAPILLA DEL CARMEN DEL SEVILLANO BARRIO DE ELCANO
}

\section{JUAN MIGUEL SÁNCHEZ'S MURAL PAINTINGS IN THE CARMEN CHAPEL OF THE SEVILLIAN ELCANO QUARTER}

\author{
JuAn Palomo-Reina \\ Universidad de Sevilla. España \\ palomore@us.es
}

\begin{abstract}
El presente trabajo tiene por objeto el estudio de las pinturas murales que realizó el pintor Juan Miguel Sánchez Fernández en 1953 para decorar el interior de la capilla de la Virgen del Carmen del barrio sevillano de Elcano. A partir del análisis de aspectos técnicos, compositivos e iconográficos, el artículo pretende incrementar el conocimiento de la obra de este pintor, rescatando del olvido esta decoración mural, de la que se ha hecho mención en publicaciones anteriores, pero que permanece prácticamente inédita y que constituye una aportación importante en el panorama pictórico sevillano de la primera mitad de siglo XX.

Palabras claves: Juan Miguel Sánchez; pintura al fresco; pintura mural: Sevilla; siglo XX.

This paper study the mural paintings that the painter Juan Miguel Sánchez Fernández achieved in 1953 in order to decorate the inside of the chapel of Our Lady of Mount Carmel in the Sevillian neighborhood of Elcano. Starting from an analysis of technical, compositional and iconographic aspects, this paper aims to increase the knowledge of this painter's work, to rescue this mural decoration from oblivion, which has been mentioned in previous publications, but remains practically unseen and is a significant contribution to the Sevillian pictorical panorama of the first half of the $20^{\text {th }}$ century.

Keywords: Juan Miguel Sánchez; fresco painting; mural painting; Seville; $20^{\text {th }}$ century.
\end{abstract}




\section{DATOS BIOGRÁFICOS Y OBRA PICTÓRICA}

Juan Miguel Sánchez Fernández nace el 1 de agosto de 1900 en El Puerto de Santa María (Cádiz). Muy pronto se inicia en el aprendizaje del dibujo y la pintura en la Academia de Santa Cecilia de El Puerto de Santa María. Con diecisiete años se traslada a Sevilla para continuar su formación en la Escuela de Artes y Oficios y en la Academia de Bellas Artes, en donde recibe la enseñanza de Virgilio Mattoni, García Ramos y Gonzalo Bilbao, entre otros. En un ambiente pictórico marcado por un eclecticismo que incluía ecos tardíos de la pintura de historia, ambientes costumbristas de un folklorismo pasado de moda, junto a una pintura luminista de influencia sorollesca, Juan Miguel Sánchez conoce en las clases de dibujo del Ateneo sevillano a Gustavo Bacarisas, autor de una obra novedosa y colorista, que va a ejercer una gran influencia en su formación, orientándolo hacia una visión más moderna de la pintura. Para mantenerse en Sevilla, trabaja la cerámica y asiste a las exposiciones de Bellas Artes que se organizaban en la capital hispalense.

En la década de los años veinte se inicia en el mundo del cartel, llegando a alcanzar grandes éxitos y diversos galardones nacionales. Su obra cartelística renueva el panorama sevillano al superar el costumbrismo imperante e introducir un aire de modernidad. Hay que destacar sus excelentes carteles de Feria de Abril y Semana Santa de 1925, 1929 y 1931, este último titulado Luz y Gracia de Sevilla, que ha sido considerado como el culmen de la cartelística sevillana por su lenguaje sintético, técnica excelente y el impacto que causa su imagen tremendamente novedosa ${ }^{1}$. Hay que destacar también su famoso cartel fauvista de la Exposición Nacional de Bellas Artes de 1926 y el cartel que realizó para la Exposición Iberoamericana de 1929.

Con respecto a su obra pictórica, su primer gran éxito lo cosecha en su primera individual en San Sebastián en 1939, dedicándose plenamente, a partir de estas fechas, a la pintura mural y de caballete. En 1945, obtiene una segunda medalla en la Exposición Nacional de Bellas Artes y, en 1948, la primera medalla con su lienzo La lección de los seises, del Museo de Bellas Artes de Sevilla.

En el ámbito de las artes suntuarias, Juan Miguel realiza una singular aportación con sus diseños originalísimos e innovadores del palio, los varales, el manto y la corona de la Virgen de los Ángeles, de la cofradía sevillana de los Negritos, de la que fue hermano y director artístico durante varios años. Por su novedad y diseño dinámico, renueva el lenguaje tradicional de la Semana Santa sevillana, dominado por la estética neobarroca.

Desempeñó cargos en distintas instituciones, como el de vicepresidente del Centro de Estudios Andaluces en 1932 y la presidencia del Ateneo Sevillano

${ }^{1}$ Cfr. GARCÍA RUIZ, José Antonio: "La técnica pictórica en la obra de Juan Miguel Sánchez Fernández”, Monografías de Arte, 2001, (CD-ROM). 
desde 1932 a 1934. Es nombrado miembro de la Real Academia de Bellas Artes de Santa Isabel de Hungría de Sevilla en 1956, en 1966 recibió la Encomienda de la Orden Civil de Alfonso X el Sabio, académico de la Real Academia de Bellas Artes de San Fernando de Madrid y vocal en la Comisión Provincial de Monumentos Histórico-Artísticos en ese mismo año.

Compaginó junto a su obra artística, su labor docente como profesor de la Escuela Superior de Bellas Artes de Santa Isabel de Hungría de Sevilla, obteniendo en 1943 la cátedra de Procedimientos Técnicos de la Pintura, que ocupó hasta su jubilación en 1970.

Juan Miguel Sánchez fallece en Sevilla en 1973.

\section{LA PINTURA MURAL EN LA OBRA DE JUAN MIGUEL SÁNCHEZ}

En su polifacética obra ocupa un puesto preferente la pintura mural. Poseedor de una sólida formación en el campo de las técnicas pictóricas, Juan Miguel destacó en el uso de la pintura al fresco y la encáustica, procedimientos cuya manipulación entrañaba grandes dificultades. Desde el siglo XIX, apenas hay pintores en Sevilla que se dediquen a la pintura mural, exceptuando a Gustavo Bacarisas (1873-1968), autor de varios murales de interés, y a Francisco Hohenleiter (1889-1971), que pintará la decoración del Teatro Coliseo. Sin embargo, la pintura mural tendrá el máximo protagonismo en el discurso pictórico de Juan Miguel Sánchez.

A nivel teórico, la preocupación por recuperar y profundizar en el conocimiento de las técnicas murales se manifiesta, de forma especial, en su discurso de ingreso en la Academia de Bellas Artes de Sevilla, en el que se describen los procesos y metodología de la técnica de la pintura al fresco, cuya práctica rescató y transmitió a sus alumnos en la Escuela de Bellas Artes hispalense. Él mismo expresa los motivos de la elección y las cualidades de esta técnica ancestral y su interés por contribuir a su enseñanza y difusión: "El hecho de haber elegido dicha modalidad pictórica para la ejecución de esta obra, y quererle dedicar unas palabras, obedece a mi decidido propósito de aprovechar cuantas ocasiones se me presenten para poner de manifiesto el gran valor plástico de la misma y las posibilidades sin límites que al practicarla nos ofrece, por creer que con la divulgación de esta antigua técnica de pintura mural, después de tanto tiempo olvidada y tantas veces desprestigiada por culpa de falsas manipulaciones de pintores desaprensivos, se hace una buena labor en bien de la pintura de nuestro momento y de la nueva generación de artistas, interesándoles por su cultivo, pues como bien dice el profesor Doerner, ninguna técnica se presta tanto como esta para las creaciones pictóricas del pensamiento moderno. $\mathrm{Y}$ es por ello por lo que me decido a contribuir 
hoy con mi modesto grano de arena, que no tiene otro valor que el de haber sido obtenido con la experiencia adquirida"2.

La obra mural más antigua de Juan Miguel Sánchez es la decoración del vestíbulo de la estación de autobuses del Prado de San Sebastián en Sevilla ${ }^{3}$, que se remonta al año 1941. Es la obra más conocida, que se compone de ocho grandes frescos, de tres metros de altura cada uno, que representan escenas relacionadas con el costumbrismo sevillano del siglo XIX. En estos murales se aprecian paisajes andaluces, con ríos, arboledas y pueblos de blancos caseríos descritos con un lenguaje más constructivo, con estructuras algo cubistas, rodeados de verdes matorrales, pitas y chumberas y árboles de tonalidades azuladas, inmersos en espacios de gran luminosidad y transparencia. Estos murales sorprenden por la modernidad de sus formas, que recuerdan un concepto constructivista a la manera de Vázquez Díaz pero con un colorido fauvista.

De 1946 es la decoración del ábside de la parroquia de la barriada cordobesa de la Electromecánica ${ }^{4}$. La pintura representa la figura central de Cristo resucitado, acompañado de la Virgen María, San Juan Evangelista y los arcángeles San Gabriel y San Rafael.

En 1949 pinta una alegoría triunfal de la Eucaristía, para decorar el coro de la iglesia sevillana de San Luis de los Franceses ${ }^{5}$, consiguiendo una perfecta integración con las pinturas existentes de Lucas de Valdés y Domingo Martínez.

Del año 1955 son los murales que decoran el vestíbulo del edificio Elcano en la avenida de las Razas ${ }^{6}$. Esta decoración se compone de tres paneles que representan tres composiciones con figuras alegóricas de Castilla y América, en el mural central, y, en los otros murales, grupos humanos con indumentaria del siglo XVI, inmersos en amplios y luminosos paisajes fluviales. Uno representa el Guadalquivir con Sevilla al fondo y otro una playa del Caribe con barcos en el mar al fondo y ricas palmeras en el primer plano. Estas pinturas tienen una gran modernidad y destaca la manera de representar los ropajes y las figuras con un concepto más plano y en el que los elementos decorativos adquieren mayor protagonismo.

${ }^{2}$ SÁNCHEZ FERNÁNDEZ, Juan Miguel: "Actualidad y enseñanzas de la pintura al fresco", Boletín de Bellas Artes, II, 1974, p. 18.

${ }^{3}$ Sobre estas pinturas cfr. FERNÁNDEZ ROJAS, Matilde: "Las pinturas murales de Juan Miguel Sánchez Fernández en la estación de autobuses del Prado de San Sebastián de Sevilla", Archivo Hispalense, 256-257, 2001, pp. 257-268.

${ }^{4}$ Sobre estas pinturas cfr. DE LA BANDA Y VARGAS, Antonio: "Los murales de la parroquia cordobesa de la Electromecánica”, Apohteca, 6, 1986, pp. 107-109.

5 DE LA BANDA Y VARGAS, Antonio: "El pintor portuense Juan Miguel Sánchez Fernández. Magisterio de Bacarisas e influencias de Vázquez Díaz", Temas de Estética y Arte, XXIV, 2010, p. 270.

${ }^{6}$ Aunque algunas publicaciones lo fechan en el año 1954, el mural aparece firmado en 1955. 
En 1956 pinta los murales de la capilla de la Real Maestranza de Caballería de Sevilla y, en 1961, lleva a cabo la decoración de la parroquia de Santa Teresa, sita en la plaza de las Moradas de Sevilla. Esta es la decoración mural de mayores dimensiones, ya que se extiende prácticamente por todos los paramentos interiores, y es la que posee una estética más novedosa fruto de su etapa de madurez. La composición, de formas muy simplificadas, con un espacio estructurado en una serie de planos, destaca por su colorido variado y por su riqueza iconográfica: filacterias con los versos del Pange Lingua y del Tantum Ergo, las figuras de los evangelistas y, en las paredes laterales, un sencillo vía crucis.

A la década de los sesenta pertenece también la decoración al fresco del vestíbulo del edificio situado en el número 18 de la sevillana calle Marqués de Paradas. Se trata de dos murales que representan escenas con figuras ataviadas a la usanza del siglo XVI sobre paisaje con el río y Sevilla al fondo. Estas pinturas tienen un aire más plano y el autor dedica especial atención a la decoración de la indumentaria y atributos de las figuras realizadas con variados colores, líneas, teniendo un aire más decorativo y desenfadado.

Con respecto a las pinturas murales desaparecidas ${ }^{7}$, hay que señalar los frescos del sevillano Bar Plata, la decoración de la fachada del Banco Vitalicio de España en la plaza Nueva, la decoración de la Horchatería Fillol, la decoración de su domicilio en la calle Matahacas y las pinturas del salón de actos del edificio de Radio Nacional de España de 1951.

\section{ANÁLISIS DE ASPECTOS COMPOSITIVOS, FORMALES Y CROMÁTICOS}

En 1953, contando con la ayuda de sus alumnos de Bellas Artes ${ }^{8}$, Juan Miguel Sánchez pinta al fresco la decoración de la capilla de la Virgen del Carmen en la barriada sevillana de Elcano. En 1966 se erige en esta capilla la parroquia de Nuestra Señora del Mar, quedando como segundo templo de la parroquia desde 1997, cuando se construye la iglesia principal en el barrio sevillano de los Bermejales. El trabajo fue encargado por la empresa nacional Elcano, posteriormente denominada Astilleros de Sevilla, que, por su intensa actividad en la construcción de navíos, generó un gran impacto económico en la ciudad y llegó a edificar en los años cincuenta la barriada de Elcano como residencia para los numerosos trabajadores de los astilleros sevillanos. El barrio de Elcano se encuentra en la zona sur de Sevilla, junto al barrio de los Bermejales.

El mural principal de la decoración que estamos estudiando ocupa el muro del presbiterio en su totalidad, desde el suelo hasta la parte alta. Tiene 9 metros

7 DE LA BANDA Y VARGAS, Antonio: "Los Murales de Juan Miguel Sánchez Fernández”, Boletín de Bellas Artes, XV, 1987, p. 116.

${ }^{8}$ El mural está firmado en el ángulo inferior derecho: "P53 de la ESBA de Santa Isabel de Hungría". 
de largo por 4,65 metros de altura en los laterales y 6,15 metros en su parte central, ya que el contorno de la parte superior es de forma triangular, por el hecho de poseer la cubierta de madera a dos aguas (Figura 1).

En su parte superior se encuentra la zona de mayor protagonismo de la composición, que aparece ocupada por la figura de la Virgen y el Niño y ocho ángeles. Esta zona, que capta la mirada de quien contempla el mural, se constituye en centro, pictóricamente potenciado por el contraste de colores y valores. En la zona que rodea a la Virgen, destacan tonalidades amarillas sobre los azules más oscuros que predominan en el cielo. También, los mástiles de las embarcaciones en primer plano apuntan, desde un extremo y otro, con su inclinación, a ese foco central, al igual que las miradas de las figuras que se encuentran en la zona inferior. De tal forma que podemos apreciar una forma triangular que ordena la composición, que tiene como vértice superior la figura de la Virgen y el Niño, y como base la línea que unifica los pies de las figuras de primer plano.

En la zona inferior del mural se encuentra el otro gran centro de la composición, ocupado por el grupo de figuras de marinos, que aparecen representados a modo de friso en primer plano. En la representación de estas figuras se aprecia una tendencia a la simplificación de las formas, y en la estructuración del espacio, el empleo de esquemas compositivos geométricos que pueden recordar la estética cubista9.

Ante esta obra, podemos percibir de manera evidente la influencia de los frescos que Vázquez Díaz pintó en 1930 en el monasterio de La Rábida, titulados Poema del Descubrimiento, en los que representa las escenas previas a la partida del puerto de Palos de la expedición que protagonizara el descubrimiento de América ${ }^{10}$. Hay similitudes en cuanto al tema -las dos narran un acontecimiento histórico del pasado protagonizado por un grupo de marinos y sus embarcaciones-, a la técnica - están realizadas al fresco- y al concepto de representación de las formas - poseen un aire constructivista-. Si bien, mientras que en las figuras de Vázquez Díaz la volumetría tiene un mayor protagonismo, en las figuras de Juan Miguel predomina una concepción algo más plana, que se va acentuando en los murales de su última época, en los cuales triunfa una concepción plana de las formas que conecta a la perfección con el lenguaje de simplificación a tintas planas de su obra cartelística.

9 VALDIVIESO, Enrique: Historia de la Pintura Sevillana. Sevilla, 1986, p. 481.

${ }_{10}$ Juan Miguel conoce con toda seguridad esta decoración mural. Además, Vázquez Díaz forma parte del tribunal que concede la cátedra a Juan Miguel Sánchez en 1943 y se conocen los elogios que hace de la obra de Juan Miguel premiada con $2^{\mathrm{a}}$ medalla en la Nacional de 1945. Posiblemente, también Juan Miguel participara en la escuela de Paisaje que fundó Vázquez Díaz en la Universidad Hispanoamericana de Santa María de la Rábida, a la que asistieron durante varios cursos profesores y alumnos de la Escuela de Bellas Artes. 
Otra cuestión a tener en cuenta en la composición es el predominio de líneas y planos verticales y oblicuos, intentando evitar, en lo posible, los planos horizontales. En este hecho se puede apreciar cierta influencia del proceder en la pintura al fresco, en la cual el mural se realiza por partes, llamadas jornadas, cuyos límites no deben percibirse, y para ello se disimulan haciéndolos coincidir con contornos de figuras o líneas de la composición; y esto se consigue con más eficacia en las uniones verticales que en las horizontales. Los límites de las jornadas coinciden con los mástiles y cuerdas de las dos embarcaciones de los extremos, y las oblicuas con los contornos de los bordes de las cubiertas de proa y popa de las mismas.

Otra característica en la manera de componer de nuestro autor es que el espacio de representación aparece bastante lleno, hay un cierto horror vacui, muy propio de la veta neobarroca de la pintura sevillana de esta época, y esto se percibe en la mayoría de sus murales.

Con respecto al color, hay que señalar el empleo de una gama cromática limitada, en la que se da un predominio de tonalidades tierra en toda la zona inferior, que contrasta con los azules del cielo y la zona central, de color amarillento, ocupada por la imagen de la Virgen y el Niño. La elección de una paleta reducida puede ser consecuencia de la experiencia de Juan Miguel en el uso de técnicas pictóricas y gráficas que imponen una limitación cromática ${ }^{11}$. Tal es el caso de la pintura al fresco, en la que se impone una selección de colores que deben ser estables en contacto con la cal, y las técnicas de impresión de carteles que se usaban en las décadas de los años veinte y treinta, que limitaban el número de tintas. Al empleo de una paleta reducida propia de la pintura al fresco, se refiere Juan Miguel cuando afirma que "una de las dificultades con que tropieza el artista al emplear por primera vez la pintura al fresco, es con el obligado reducido número de colores que puede emplear en esta técnica, acostumbrado a una paleta amplia de tonalidades" ${ }^{\prime 2}$. Sin embargo, esta imposición tiene consecuencias positivas, que confirma con su experiencia como pintor: "Pero creo que precisamente a esa limitada y sobria gama de colores se debe gran parte del encanto de esta modalidad pictórica. Para ello debe tenerse siempre presente, según mi experiencia, que cuanto mayor es un mural, más necesaria se hace la simplificación en el color y más perjudicial resulta en él el abuso de los tonos enteros. Así pues, mi paleta para esta técnica procuro que sea lo más sobria posible y por tanto, solo utilizo, generalmente, los colores que doy a continuación: Blanco, la cal; negro, de marfil; tierras, natural y quemada; ocre amarillo, claro y oscuro; rojo, de óxido de hierro; rojo tierra de Sevilla; verde, de óxido de cromo; verde de óxido de cobalto, y azul, de óxido de cobalto" ${ }^{\prime 3}$.

${ }^{11}$ GARCÍA RUIZ, José Antonio: "La técnica pictórica de...”, op. cit.

12 SÁNCHEZ FERNÁNDEZ, Juan Miguel: "Actualidad y enseñanzas de la...”, op. cit., p. 28.

13 Ibidem.

LABORATORIO DE ARTE 30 (2018), pp. 439-456, ISSN 1130-5762 e-ISSN 2253-8305 - DOI http://dx.doi.org/10.12795/LA.2018.i30.24 
Esta experiencia en el uso de una serie de colores restringida en el fresco influye también en su pintura de caballete. A este respecto afirma el pintor García Ruiz, alumno de Juan Miguel: "Esta misma paleta de colores simple empleó también para su pintura de caballete, una gama de color simple y exenta de colores primarios, en la cual, él había encontrado una especial belleza y puedo asegurar que las veces que pude visitar su estudio, observé que los colores que utilizaba para la pintura de caballete son los mismos que describe para la pintura al fresco"14.

\section{LA TÉCNICA DE LA PINTURA AL FRESCO EN EL MURAL DE LA CAPILLA DE ELCANO}

Juan Miguel tiene especial predilección por la pintura al fresco. En su discurso, tras señalar las exigencias y grandes dificultades de esta técnica, describe sus virtudes y excelencias: "Pero los resultados que con esta técnica se obtienen compensan sobradamente nuestros desvelos, pues la fuerza plástica de este procedimiento no puede superarse y ni siquiera igualarse con ningún otro de los conocidos, ya que se reúnen en él las cualidades más características de todos los demás. Posee de la acuarela su frescura y fluida transparencia, del temple y el pastel el mate impecable y etéreo, y de la encáustica y el óleo su extraordinaria fuerza plástica, unido todo ello a otras cualidades que tanto le caracterizan, como son su empaque sobrio y grave, impuesto por el limitado número de colores y condición de los mismos, que pueden emplearse, y su solidez indiscutible a través del tiempo" 15 .

Para la ejecución del mural de Elcano, se aplicaron varias capas de mortero de cal y arena. La primera, llamada repellado, se compone de tres partes de arena de río y una de pasta de cal. La segunda capa, que se aplica sobre la anterior cuando está seca, se llamada revoque, y está constituida por el mismo mortero pero algo más espeso, debiendo quedar con una textura rugosa en superficie y gran porosidad para que se produzca una fuerte trabazón entre las primeras y siguientes capas de enlucido y estucado. Estas dos capas anteriores deben tener un grosor de un centímetro cada una. Sobre esta última capa se extiende con fratás y palustre el enlucido, que es otro mortero compuesto por dos partes de polvo de mármol y una de cal y su grosor debe oscilar entre tres y cinco milímetros. La superficie del enlucido debe quedar apretada y alisada pero con cierta rugosidad. Tras unos minutos, se aplica la última capa formada por un mortero de cal y polvo de mármol a partes iguales.

${ }^{14}$ GARCÍA RUIZ, José Antonio: "La técnica pictórica de...", op. cit.

15 SÁNCHEZ FERNÁNDEZ, Juan Miguel: "Actualidad y enseñanzas de la...”, op. cit., p. 23. 
Antes de aplicar el color, hay que traspasar las líneas del dibujo a la pared por método inciso ${ }^{16}$ o por estarcido. Juan Miguel se inclina por el segundo, que se reconoce a simple vista cuando al observar el mural a poca distancia se aprecian los puntos que forman las líneas del dibujo en el mural terminado. Juan Miguel describe con pormenores el método, que, según él, daña menos el mortero húmedo, y que consiste en dibujar la composición en un papel con el tamaño definitivo, agujerear las líneas, tender el papel sobre el muro y con una muñequilla de carbón molido presionar para que el carbón penetre por los orificios del papel y queden marcados los puntos de las líneas del dibujo en el enlucido.

A continuación, se unen estos puntos con un trazo ágil y expresivo, siempre con pincel de pelo blando y posteriormente se inicia la aplicación del color empezando por los tonos claros, seguidos de los intermedios hasta llegar a los más oscuros. Juan Miguel Sánchez traza perfectamente todas las líneas del dibujo con pincel fino, y estas líneas permanecen visibles en el mural terminado, sin ser cubiertas totalmente por las zonas de color, contribuyendo así al refuerzo de las formas.

El trazo y las pinceladas suelen ser directas, el color se aplica de una vez, alla prima, evitando hacer correcciones y superponer otras capas de pintura. Por esto, su manejo exige una gran destreza y habilidad. Juan Miguel considera importante esta forma de trabajar y nos deja constancia por escrito cuando habla de la técnica del genial pintor fresquista Fra Angélico: "Cuenta Jorge Vasari, en su obra Vida de grandes artistas, al hablar de Fray Juan Angélico, que tenía por costumbre no retocar ni corregir ninguna obra suya, sino dejarla siempre tal y como había quedado la primera vez, por creer, según decía, que tal era la voluntad de Dios. Con lo que nos hace ver claramente que gran parte de la belleza de los célebres frescos del fraile pintor se debe, sin duda alguna, a su santa costumbre de una parte, y de otra a los profundos conocimientos técnicos que poseía. A esta difícil facilidad de ver, sentir y hacer que está siempre presente en todas sus creaciones y son cualidades fundamentales para poder emplear con éxito el procedimiento pictórico mural de que hemos tratado" 17 .

Aunque el mural está pintado al fresco, hay zonas en las que se han incluido retoques $a \operatorname{secco}^{18}$, con temple y óleo. Esto se aprecia sobre todo en la zona del cielo y las nubes cercanas a la figura de la Virgen, en donde se ha conseguido una

${ }^{16}$ Consiste en presionar sobre las líneas del dibujo que previamente se ha trazado en un papel a tamaño real, consiguiendo que estas queden marcadas en la última capa del enlucido, que está húmedo.

${ }^{17}$ SÁNCHEZ FERNÁNDEZ, Juan Miguel: “Actualidad y enseñanzas de la...”, op. cit., p. 30.

${ }_{18}$ A secco es un término italiano que se usa para denominar los retoques con otra técnica sobre un mural pintado al fresco, una vez que el mortero ha fraguado, y por lo tanto la superficie del mural está ya seca. 
superficie muy redondeada, con una sutil gradación que parece haber sido hecha aplicando la pintura con esponja. También hemos observado el empleo de retoques con óleo aplicado con una consistencia pastosa en los blancos y algunas tonalidades tierras y azules oscuros de las vestiduras de los marineros.

A pesar de que toda pintura mural exige un estudio previo a la realización definitiva sobre el muro, para lo cual es necesario pintar el cartón ${ }^{19}$, Juan Miguel, tras estudiar perfectamente la composición y las líneas del dibujo, prefiere dejar algo a la espontaneidad del pintor. Considera innecesario y contraproducente la costumbre de aplicar el color y terminar los cartones como si fuera la pintura definitiva. Este hecho, dice el autor, "tiende a cansar el gusto del pintor y pone trabas a la espontaneidad; cierra materialmente el acceso a ese ilimitado campo de acción que siempre debe estar abierto a la inspiración del artista durante la realización de su obra" 20 .

Para los cartones, dibuja a carbón sobre papel de color marrón o gris las líneas de toda la composición ya concluida, y, si el tema lo requiere, ilumina las luces con blanco al pastel. Por último, señalar que si el cartón contiene solo el dibujo y alguna insinuación de luces, es indispensable para la ejecución de cualquier pintura mural la realización de un boceto previo a escala que debe estar muy terminado y en él se hayan resuelto todos los problemas de forma, color y composición, que permita enfrentarse con éxito a la obra definitiva sobre el muro.

\section{LA ICONOGRAFÍA DE ESTA DECORACIÓN MURAL}

Desde el punto de vista de la iconografía, la decoración mural de esta iglesia se puede dividir en dos partes. La primera comprende las pinturas del arco y paramentos laterales que flanquean el presbiterio y las jambas de las ventanas del edificio, que contienen motivos alegóricos de la Eucaristía empleados ya en otros murales religiosos por Juan Miguel ${ }^{21}$.

En la zona central sobre el arco aparecen representadas espigas y racimos de uva (Figura 2), junto con una filacteria que contiene la primera estrofa del sublime himno eucarístico de Santo Tomás Pange lingua: "PANGE, LINGUA,

${ }^{19}$ Se llama cartón al dibujo definitivo a tamaño real que contiene la composición formal perfectamente terminada para ser calcado al muro por distintos métodos.

${ }^{20}$ SÁNCHEZ FERNÁNDEZ, Juan Miguel: “Actualidad y enseñanzas de la...”, op. cit., p. 27.

${ }_{21}$ Aparece en los murales que realizó en Sevilla para la parroquia de Santa Teresa, capilla de Elcano, iglesia de San Luis, capilla de la Maestranza y en Córdoba para la parroquia de la barriada de la Electromecánica. 


\section{GLORIOSI/ CORPORIS MYSTERIUM/ SANGUINISQUE PRETIOSI,/ QUEM IN MUNDI PRETIUM"22.}

Los paramentos laterales están decorados con sendos ángeles, vestidos con túnicas rojas y estrellas amarillas, con alas doradas y rubia cabellera, que sostienen, con un efecto ilusionista, unos soportes de forja de los que penden sendas cadenas con anclas de cuatro brazos ${ }^{23}$, en cuyos extremos se sitúan las lámparas que iluminan la nave (Figura 3). El tratamiento de estas figuras es más colorista y decorativo, y sus ropajes están hechos con trazos y líneas muy dinámicas.

Esta primera decoración es la antesala de la zona del santuario en donde se encuentra el altar actual y el primitivo, de madera y que posee atributos marineros, situado a escasos centímetros del muro del presbiterio en el que está pintado el mural que corresponde a la segunda parte de la iconografía. En este mural se representa la composición con figuras junto al río en cuya parte superior se puede contemplar el rompimiento de gloria, con la figura de María sedente, en su advocación de la Virgen del Carmen, con el Niño Dios en sus brazos.

La Virgen aparece ataviada con el hábito carmelita: túnica marrón y capa blanca. Y en la mano derecha porta el santo escapulario, que, según la tradición, la Virgen entregó a San Simón Stock en una aparición el día 16 de julio de 1251. El Niño bendice con su mano derecha y sostiene el escapulario con la izquierda. La figura de la Virgen y el Niño son de una gran belleza y ternura. El tratamiento de su manto y vestido introduce un gran dinamismo que le dan un aire muy novedoso y alegre.

Rodeando a la Virgen y al Niño, en la parte superior vemos tres cabezas de querubines y cuatro a sus pies. A derecha e izquierda, dos ángeles niños que sostienen una filacteria en la que aparecen las primeras palabras de un himno latino que se canta en las vísperas de las fiestas marianas: "AVE MARIS STELLA. DEI MATER ALMA"24. Uno lleva en la otra mano rosas blancas y el otro un pequeño barquito de vela (Figura 4 ).

En la mitad inferior del mural aparece un grupo de figuras dispuestas a modo de friso frente a un paisaje del río Guadalquivir con una vista de Sevilla al fondo.

La pintura representa un acontecimiento histórico de gran importancia para la historia de la humanidad: la llegada al puerto de Sevilla de la expedición que daría la primera vuelta al mundo. La nao Victoria, capitaneada por Juan Sebastián Elcano, con diecisiete supervivientes más, fue el único barco de los cinco que formaban la expedición que regresó a España, convirtiéndose en la primera embarcación en circunnavegar la Tierra. De los doscientos treinta y cuatro marineros

22 "Cante la voz del Cuerpo más glorioso el misterio sublime y elevado, y la Sangre preciosa, que amoroso, en rescate del mundo ha derramado".

${ }^{23}$ Este tipo de ancla aparece también pintada en el mural central del hall del edificio Elcano.

24 "Salve, Estrella del Mar. Madre de Dios excelsa". 
que empezaron el viaje, el 10 de agosto de 1519, solo dieciocho llegaron con vida al puerto de Sevilla.

La pintura representa a Juan Sebastián Elcano y la tripulación de la nao Victoria, tras desembarcar en Sevilla el 8 de septiembre de 1522, día de la Natividad de la Virgen, portando grandes cirios encendidos, antes de iniciar la procesión de acción de gracias a las iglesias de Nuestra Señora de la Victoria en el convento sevillano de los mínimos del barrio de Triana y a Nuestra Señora de la Antigua en la catedral de Sevilla, por haber podido completar la primera vuelta al mundo ${ }^{25}$. En la narración de este acontecimiento histórico tan emotivo, Juan Miguel incluye a la Virgen del Carmen con el Niño como patrona y protectora de los hombres de la mar, en un cielo que se abre, ante los rostros llenos de fervor de los supervivientes de la primera vuelta al mundo.

Aunque la pintura representa una escena histórica, su autor no cae en los arqueologismos que tanto perjudicaron a la pintura de historia del siglo XIX. Sus figuras tienen un aire de naturalidad y gran viveza, quedando los detalles anecdóticos sobre indumentaria y otras cuestiones en un segundo plano (Figura 5). Juan Miguel supo incorporar un cierto aire de modernidad, al conjugar temas como este, y en el resto de sus obras otras temáticas tradicionales sevillanas, con un aire naturalista y una concepción constructiva de las formas ${ }^{26}$.

El grupo se compone de veintiséis figuras en el primer plano, de dimensiones algo mayores del tamaño natural que, al poseer gran variedad de formas, gestos y posiciones, contribuyen, junto con las trece figuras más pequeñas que aparecen en los barcos y barcas del fondo, a dar a la escena marinera un ambiente muy dinámico. Las figuras aparecen agrupadas en dos zonas: la de la izquierda con catorce figuras, entre las que destaca en el centro el que parece ser Juan Sebastián Elcano, arrodillado y mirando a la Virgen (Figura 6) y la de la derecha con doce, entre los que se encuentran dos frailes franciscanos y otros caballeros con capa y sombreros de la época (Figura 7). En total son los dieciocho marineros de la tripulación de la nao Victoria, cinco caballeros, dos frailes y un personaje de larga barca con una túnica y cabeza cubierta. Tres aparecen en actitud orante con las manos juntas, siete arrodillados, catorce figuras portan cirios en las manos, doce levantan la mirada para contemplar a la Virgen y dos miran al frente, siguiendo el recurso tan utilizado en la historia de la pintura que busca introducir en la escena representada a la persona que contempla el mural.

En el primer plano, enmarcando el grupo se encuentran la proa de la nao Victoria a la derecha y la popa de otra embarcación, cuyos mástiles, maromas y velas estructuran la composición de esta escena que se desarrolla en el célebre puerto de las Muelas sobre el Guadalquivir, situado antaño en donde hoy se encuentra

${ }^{25}$ Otra pintura que representa este tema singular es el óleo pintado en 1929 por Elías Salaverría Inchaurrandieta (1883-1952) que se encuentra en el Museo Naval de Madrid.

${ }^{26}$ GUASH, Ana: 40 años de pintura en Sevilla (1940-1980). Sevilla, 1981, p. 15. 
el puente de San Telmo, a la entrada del barrio de los Remedios. Sobre las aguas del Guadalquivir cinco barcos más de mayor tamaño y cuatro barcas con remeros animan el río. Al fondo se contempla una vista de la Sevilla del siglo XVI, en la cual destaca, sobre el caserío blanco de la ciudad, la catedral y la Giralda, culminada con una espadaña en vez del cuerpo de campanas y el Giraldillo, que fueron construidos con posterioridad al acontecimiento que narra la escena ${ }^{27}$. Así mismo, observamos la Torre del Oro sin el remate que posee actualmente y la muralla con dos de sus puertas junto la explanada del Arenal.

El mural de la capilla del Carmen de Elcano nos presenta una escena profundamente dinámica en la que alternan y se comunican con total naturalidad dos ámbitos. Una realidad natural en la tierra, descrita con multitud de detalles -marinos con variadas posturas y expresiones, embarcaciones que animan las aguas del Guadalquivir con sus mástiles y velas y la vista de Sevilla- y otra realidad sobrenatural en el cielo, con la Virgen, el Niño y unos ángeles pequeños que, con sus rostros risueños, nos hacen cercano el misterio. Todo transmite dinamismo, alegría y novedad, notas singulares de la pintura de Juan Miguel que, tanto en sus murales, como en sus carteles o en los diseños que realizó para las artes suntuarias, pretendió, conservando lo mejor de la tradición sevillana, aportar aires nuevos que renovaran amablemente el panorama pictórico de la Sevilla de su época.

Sirva el presente artículo para recuperar la figura y la obra pictórica de Juan Miguel Sánchez Fernández, pintor que gozó de gran reconocimiento y renombre en su tiempo y en cuya obra he querido profundizar para poner de relieve la aportación que esta supone a la historia de la pintura mural sevillana.

Fecha de recepción: 30 de septiembre de 2017

Fecha de aceptación: 12 de abril de 2018

${ }^{27}$ Pudiera haberse documentado Juan Miguel Sánchez en las famosas ilustraciones de Alejandro Guichot y Sierra Los tres principales estados de la Giralda de Sevilla, publicado en 1910, que a su vez se basaba en varias fuentes entre las que está la pintura de 1555 de Hernando de Esturmio del retablo de la capilla de los Evangelistas de la catedral hispalense, que representa a las Santas Justa y Rufina con la Giralda en el centro, ya que tiene mucha similitud. 


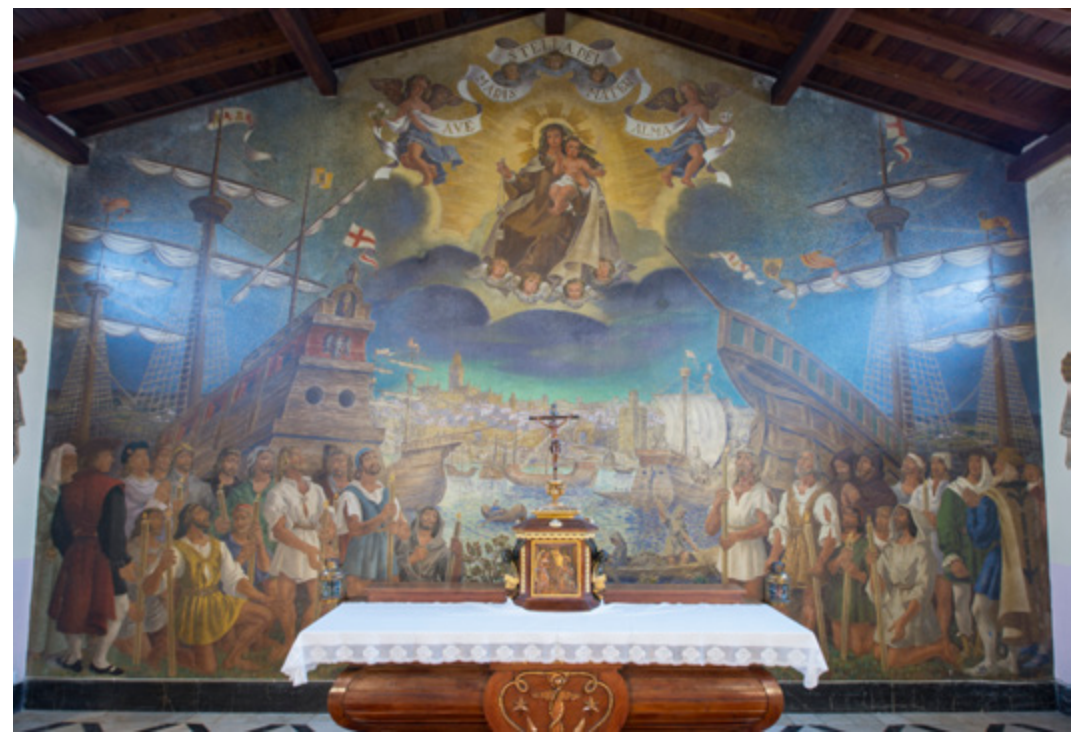

Figura 1. Juan Miguel Sánchez, La Virgen del Carmen y la tripulación de la nao Victoria a su llegada al puerto de Sevilla, 1953, pintura al fresco en el muro del presbiterio, capilla del Carmen del barrio de Elcano, Sevilla.

Foto: Fernando Soriano Arias.

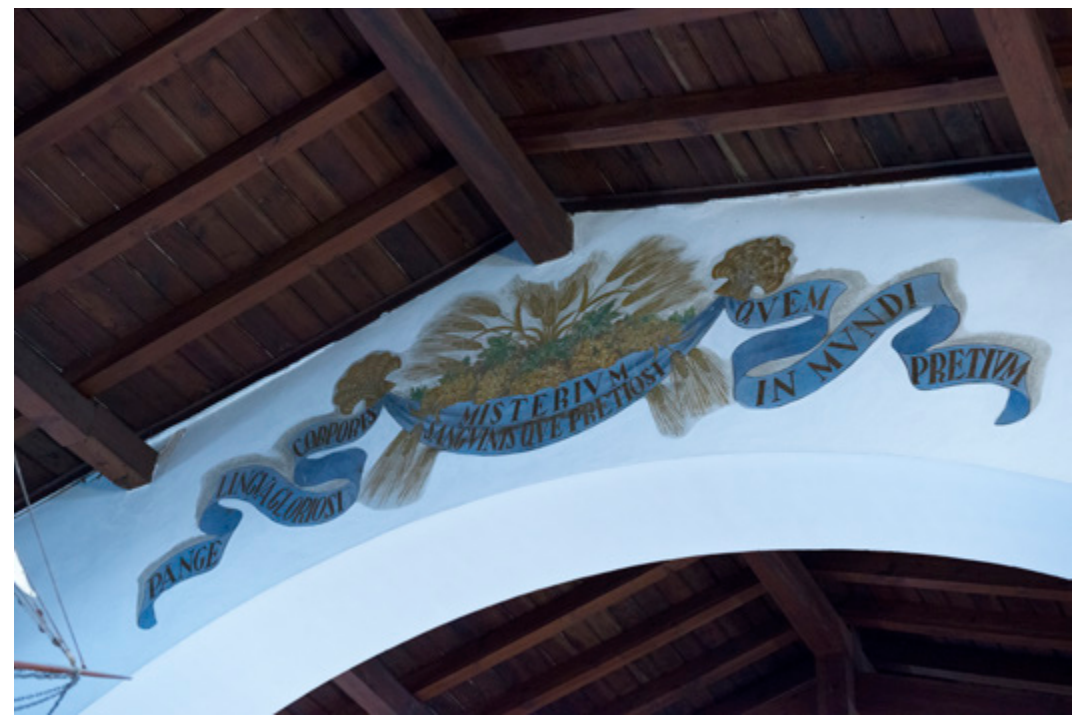

Figura 2. Juan Miguel Sánchez, Alegoría a la Eucaristía, 1953, pintura al fresco en el arco de acceso al presbiterio, capilla del Carmen del barrio de Elcano, Sevilla. Foto: Fernando Soriano Arias. 


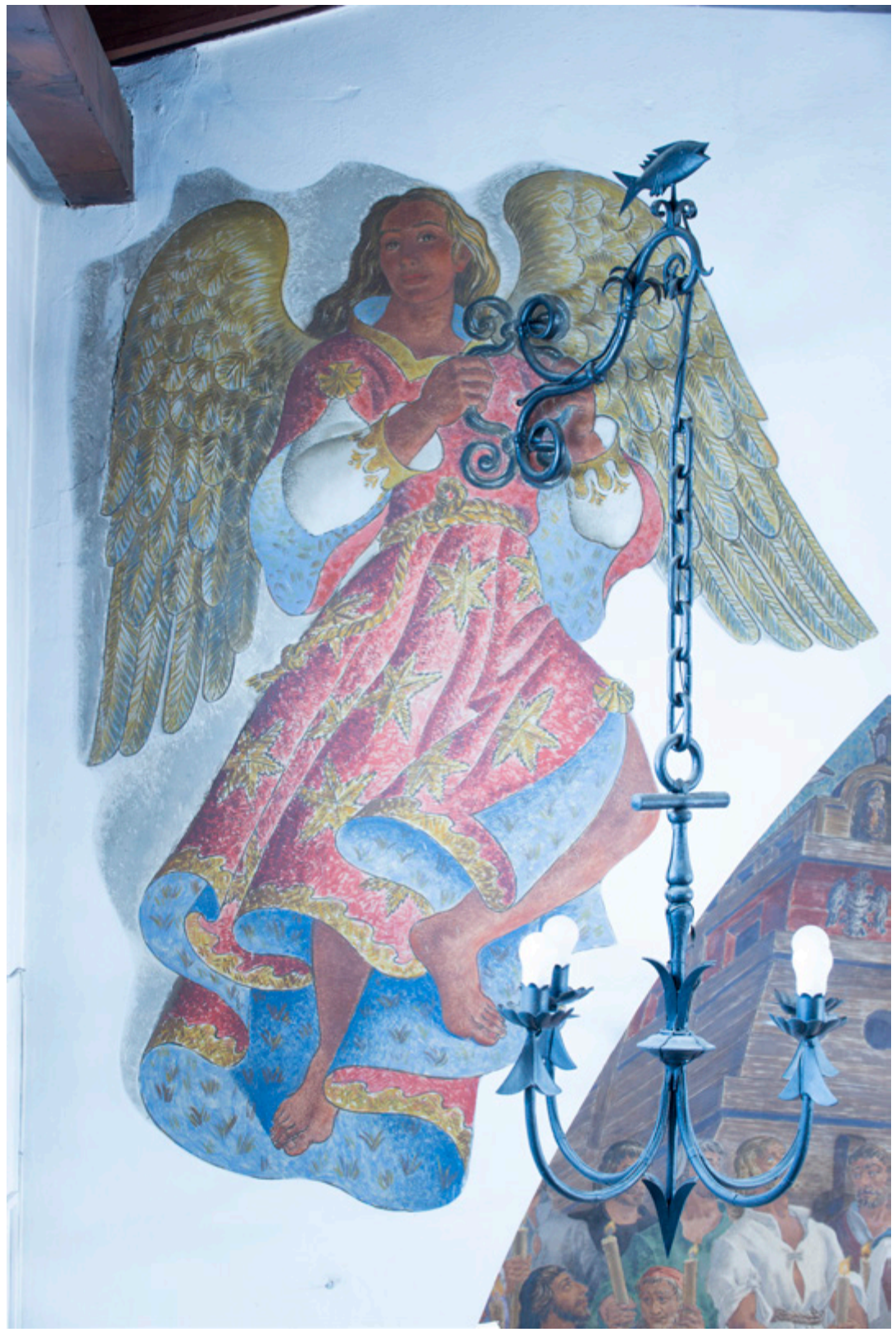

Figura 3. Juan Miguel Sánchez, Ángel con lámpara, 1953, pintura al fresco en el paramento lateral izquierdo, capilla del Carmen del barrio de Elcano, Sevilla. Foto: Fernando Soriano Arias. 


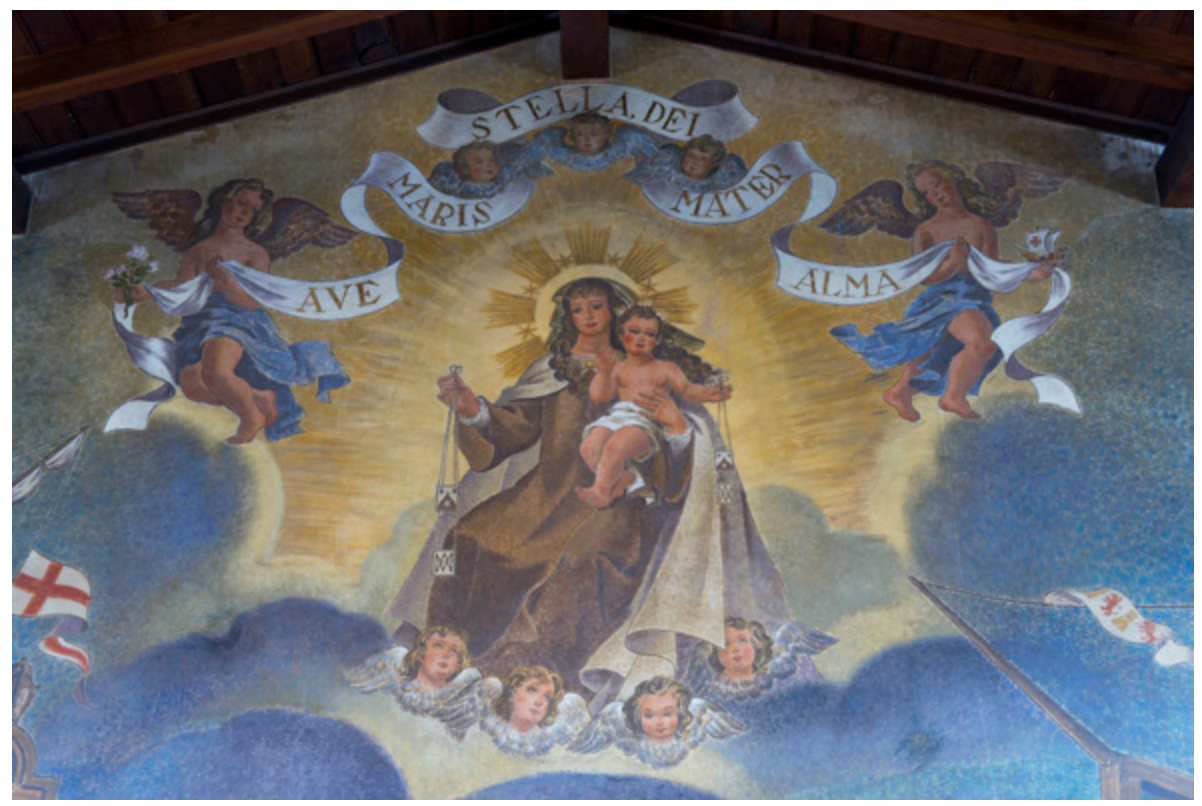

Figura 4. Juan Miguel Sánchez, Virgen del Carmen con el Niño y ángeles, 1953, detalle de la zona superior del muro del presbiterio, capilla del Carmen del barrio de Elcano, Sevilla. Foto: Fernando Soriano Arias. 


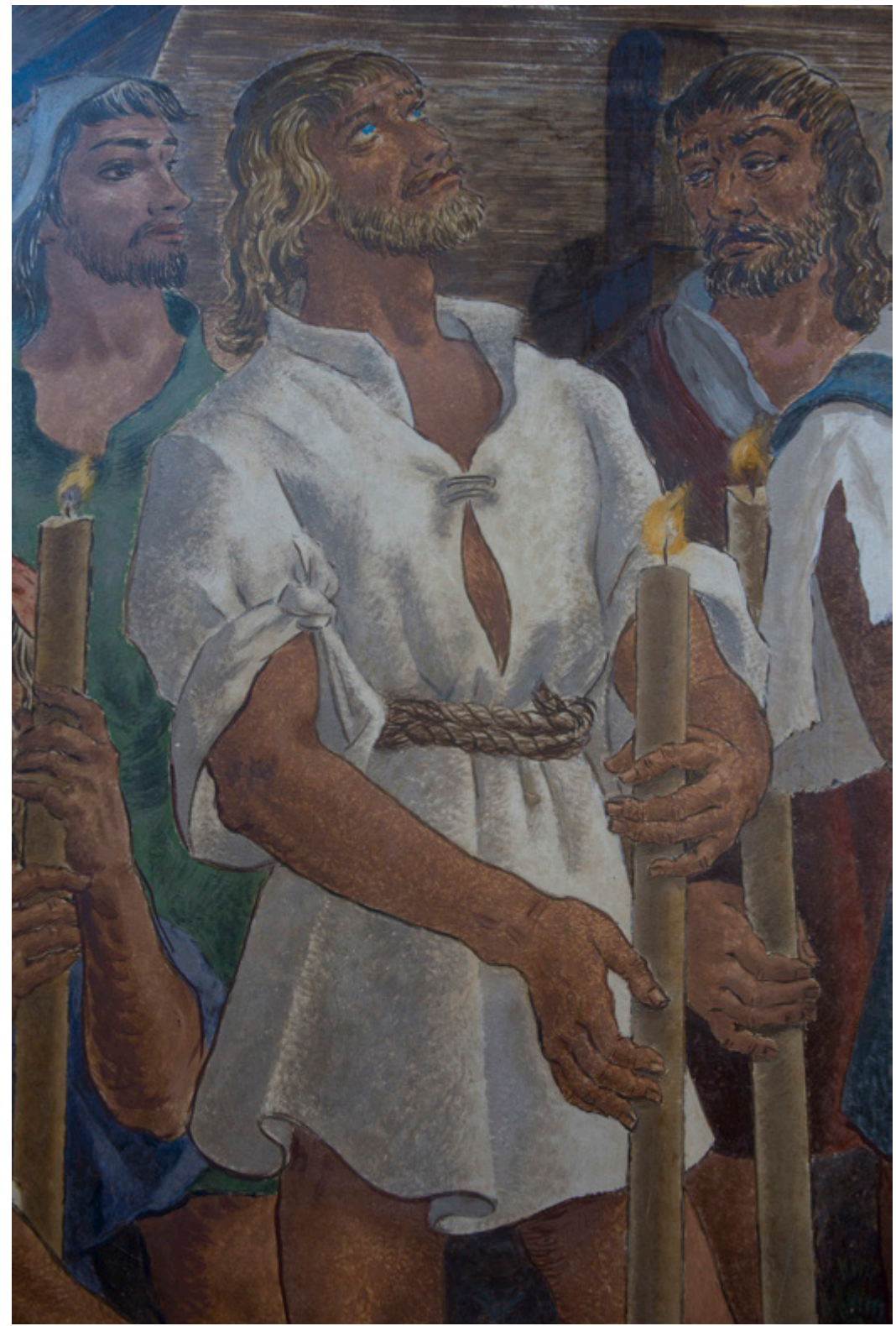

Figura 5. Juan Miguel Sánchez, Personaje, 1953, detalle del grupo de la izquierda del muro del presbiterio, capilla del Carmen del barrio de Elcano, Sevilla. Foto: Fernando Soriano Arias. 


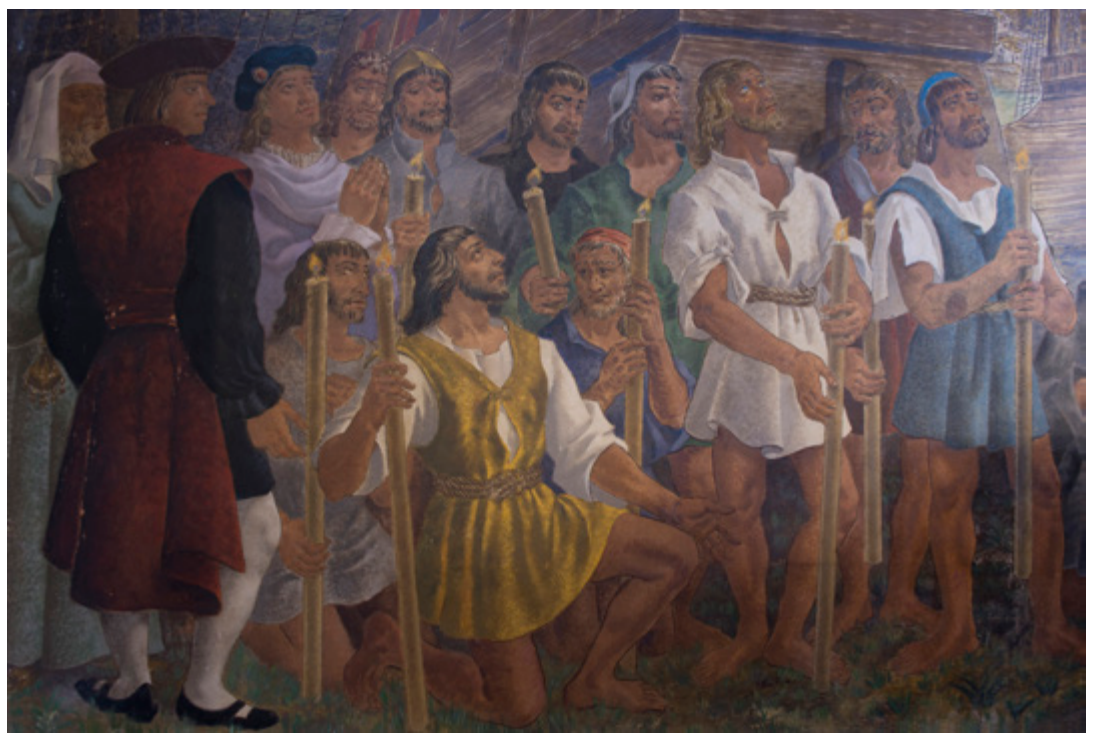

Figura 6. Juan Miguel Sánchez, Grupo de tripulantes de la nao Victoria a la izquierda (detalle), 1953, pintura al fresco, capilla del Carmen del barrio de Elcano, Sevilla. Foto: Fernando Soriano Arias.

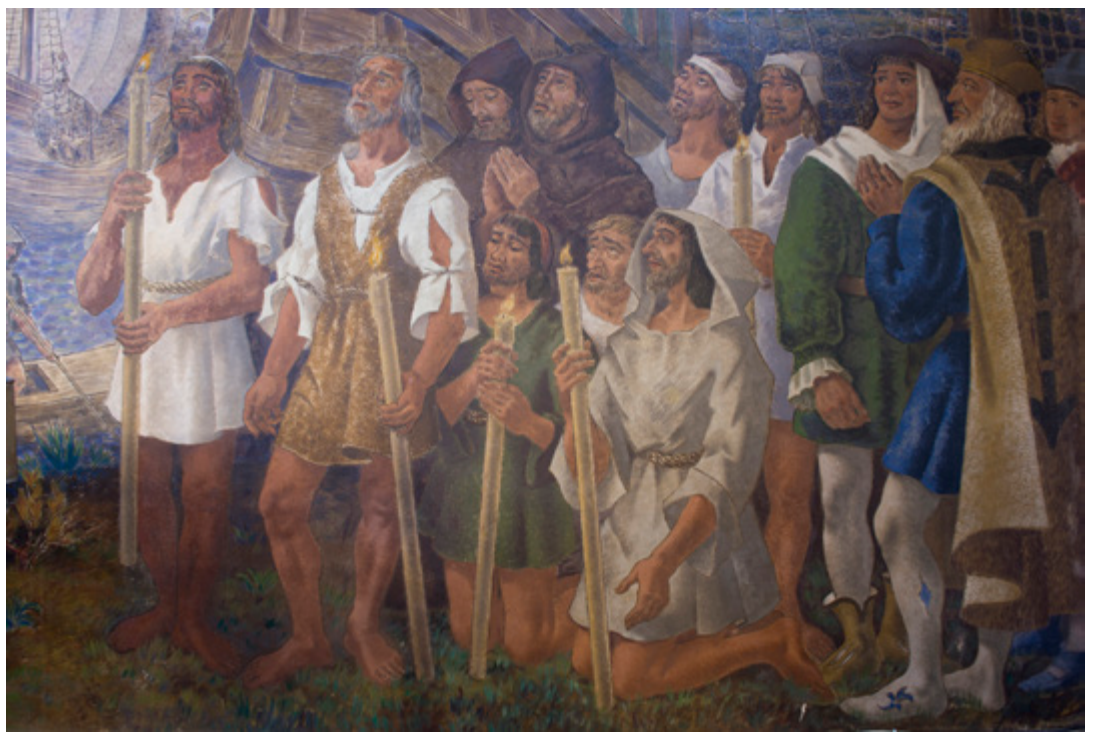

Figura 7. Juan Miguel Sánchez, Grupo de tripulantes de la nao Victoria a la derecha (detalle), 1953, pintura al fresco, capilla del Carmen del barrio de Elcano, Sevilla. Foto: Fernando Soriano Arias. 